\title{
Inhibition of cAMP responsive element binding protein in striatal neurons enhances approach and avoidance responses toward morphine- and morphine withdrawal-related cues
}

\author{
Carles Sanchis-Segura ${ }^{1 *}$, Dragana Jancic ${ }^{2}$, Maria Jimenez-Minchan ${ }^{2}$ and Angel Barco ${ }^{2 *}$ \\ Area de Psicobiología, Universitat Jaume I de Castello, Castello, Spain \\ 2 Instituto de Neurociencias de Alicante, Universidad Miguel Hernández-Consejo Superior de Investigaciones Cientificas, Alicante, Spain
}

\section{Edited by:}

Serge Laroche, CNRS and Université

Paris Sud, France

\section{Reviewed by:}

Bruno Bontempi, CNRS and Université de Bordeaux, France

Martine Ammassari-Teule, Consiglio

Nazionale delle Ricerche, Italy

*Correspondence:

Angel Barco, Instituto de

Neurociencias de Alicante, UMH-CSIC,

Campus de Sant Joan, Apt. 18, Sant

Joan d'Alacant 03550, Alicante, Spain.

e-mail: abarco@umh.es;

Carles Sanchis-Segura, Area de

Psicobiologia, Universitat Jaume I

de Castello, 12071 Castello, Spain.

e-mail: csanchis@psb.uji.es
To investigate the role of CAMP responsive element binding protein (CREB)-dependent gene expression in morphine induced behaviors, we examined bitransgenic mice expressing a dominant and strong inhibitor of the CREB family of transcription factors, A-CREB, in striatal neurons in a regulatable manner. The expression of $A-C R E B$ in the striatum enhanced both morphine-induced conditioned place preference and morphine withdrawal-induced conditioned place avoidance. Our experiments thereby support a role for CREB in striatal neurons regulating approach and avoidance responses toward drug-related cues.

Keywords: CREB, morphine, addiction, place preference, striatum

\section{INTRODUCTION}

In their attempt to adapt to the environment and survive, individuals rapidly learn to approach or avoid biologically relevant stimuli as well as the cues and contexts that predict their appearance (Domjan, 2005). As subserving learning, that is, changes in perception, interpretation and response to relevant stimuli, neural plasticity is a main determinant of animal's behaviors and of their adaptive capabilities. Through its regulatory role on gene expression, transcription factors are key elements of neural plasticity and its consequences on behavior (McClung and Nestler, 2008).

The cAMP responsive element (CRE)-binding protein, $\mathrm{CREB}$, is a constitutively expressed transcription factor that becomes activated by phosphorylation through several signaling cascades and represents a convergence point through which incoming information from several intracellular pathways can be integrated and translated in different patterns of gene expression (Mayr and Montminy, 2001). Accordingly, CREB has been associated with neuroplasticity processes underlying different forms of learning and memory and several psychiatric disorders, including the cellular and molecular changes induced by drugs of abuse (Carlezon et al., 2005). In this regard, it has been shown that psychostimulants and opiates increase CREB phosphorylation and CRE-mediated gene expression in specific neuronal populations (Konradi et al., 1994; Hyman et al., 1995; Shaw-Lutchman et al., 2003; Walters et al., 2003; Carlezon et al., 2005; Olson et al., 2005). The behavioral significance of these molecular adaptations is complex and seems to differ notably among different brain areas (Carlezon et al., 2005). Indeed, CREB-related neuroplasticity has been previously related to drug reinforcement, tolerance, and withdrawal (Nestler, 2004; Carlezon et al., 2005).
Here, we assess the role of this transcription factor in morphineinduced behaviors and striatal function using a new strain of bitransgenic mice that express A-CREB, a dominant and strong inhibitor of the CREB family of transcription factors in striatal neurons in a regulatable and highly restricted manner. Our experiments confirm previous findings and support a role for CREB-dependent gene expression in GABAergic striatal neurons regulating approach/ avoidance behaviors toward cues associated to appetitive/aversive consequences derived from chronic drug exposure.

\section{MATERIALS AND METHODS}

\section{GENERATION AND MAINTENANCE OF TRANSGENIC MICE}

Several lines of transgenic mice expressing A-CREB under the control of tet $O$ promoter were generated by microinjection and backcrossed to C57BL/6J. We have previously described in detail (Jancic et al., 2009) the bitransgenic mice resulting of the crossing of $p$ CaMKII-tTA mice line B (Mayford et al., 1996) and tetO-A-CREB line AC95 (ACREB-FB in Figure 1A), which showed the highest level of expression in the hippocampus among the different founder obtained by microinjection. In this study, we investigated the phenotype of bitransgenic animals that resulted of the crossing of $p C a M K I I-\mathrm{tTA}$ mice line $\mathrm{B}$ and tetO-A-CREB line $\mathrm{AC} 100$, which express the transgene exclusively in the striatum. We refer to this bitransgenic strain as ACREB-St mice. In all our experiments, we used as control littermate mice carrying either $p C a M K I I-\mathrm{tTA}$, tetO-A-CREB or none transgene. For reversal experiments, doxycycline (dox) was administrated at $40 \mathrm{mg} / \mathrm{kg}$ of food and added at the indicated times. Experiments were performed in adult male mice (2- to 4-month-old). All mice were 
maintained and bred under standard conditions, consistent with national guidelines and approved by the Institutional Animal Care and Use Committees.

\section{DRUGS}

Morphine sulfate and naloxone hydrochloride were purchased from Sigma Aldrich Química S. A. (Madrid, Spain). Drugs were dissolved in a saline $(0.9 \% \mathrm{w} / \mathrm{v})$ solution and injected intraperitoneally (i.p.). The same volume of vehicle was administered to control groups.

\section{BASAL BEHAVIOR}

An initial behavioral characterization was performed in a separate cohort of ACREB-St $(N=9)$ and control $(N=7)$ littermate mice. Open field locomotion: Mice locomotion in the open field was measured in a single testing session (duration: $20 \mathrm{~min}$ ) conducted in a room softly illuminated. The open-field chamber consisted of a clear glass cylinder $25 \mathrm{~cm}$ in diameter and $30 \mathrm{~cm}$ high. Locomotor activity was registered by a computerized video tracking system (SMART, Panlab S.L; Barcelona, Spain) and calculated as travelled distance (centimeter). Novel cage test: Reactivity to a novel environment was tested by assessing the number of rearing episodes in a single exposure (duration: $5 \mathrm{~min}$ ) to a new environment. The new environment was a standard type II macrolon cage with a thin layer of bedding material (Sanchis-Segura et al., 2006). Anxiety-like behavior: Anxiety-like behavior was measured by means of two well-standardized tests: The elevated plus maze and the dark-light box.

\section{MORPHINE RELATED BEHAVIORS}

Morphine-induced conditioned place preference (CPP): CPP was assessed using fourblackacrylic chambers $(30 \mathrm{~cm} \times 15 \mathrm{~cm} \times 20 \mathrm{~cm})$ contained in a dimly-illuminated and sound attenuated enclosure. No divider was used in the chambers so animals had access to the entire box. Tactile cues (interchangeable grid and hole floors) were used as conditioned stimuli (CS). The behavioral procedure had three phases: (1) A habituation session was implemented by placing each animal in the CPP chambers (floor divided with half grid, half holes) for $15 \mathrm{~min}$. (2) Mice were examined in eight trials (60-min duration; one trial per day), corresponding to four morphine/CS+ pairings and four saline/CS- pairings. The use of both floor types as CS+ or CS- was counterbalanced among groups using an unbiased CPP design. Morphine $(20 \mathrm{mg} / \mathrm{kg})$ or saline was administered immediately before placing the mice into the conditioning chambers prepared with the corresponding CS+ or CS- floor. CS+ and CS- pairing sessions were alternated following an $\mathrm{ABABAB}$ sequence with all animals receiving first a CS+ session. (3) A single preference test (duration: $15 \mathrm{~min}$ ) was conducted the day after the last conditioning session. In this case the floor of the conditioning chambers was divided (half grid, half holes) and the time spent in each floor was assessed by a video-tracking system (SMART, Panlab S.L; Barcelona, Spain). As described in the statistics section, the percent of time spent in the CS+ compartment was the preference index used in this experiment. Opioid dependence induction: Opioid dependence was induced by repeated injections of morphine based on the procedure described by (Maldonado et al., 1996). Mice received morphine injections twice a day (9 AM and 9 PM) with progressively increasing doses $(20,40,60,80$, and $100 \mathrm{mg} / \mathrm{kg}$, i.p. $)$ in their home cages for five consecutive days. On the 6th day, mice were treated with a single morphine (100 mg/kg, i.p.; 9 AM) injection. Separate groups of mice exposed to this dependence-induction drug schedule were used for assessing morphine withdrawal signs or morphine withdrawal-induced conditioned place avoidance (CPA). Expression of morphine withdrawal signs: Morphine dependence was induced by the procedure described above. Ninety minutes after the last morphine injection (day 6 ), mice were individually placed in cubic metacrylate boxes $(40 \mathrm{~cm} \times 40 \mathrm{~cm} \times 40 \mathrm{~cm})$. Fifteen minutes later, morphine withdrawal was precipitated by a subcutaneous naloxone injection $(0.1 \mathrm{mg} / \mathrm{kg})$ and mice behavior was videotaped for additional $15 \mathrm{~min}$. Several signs of the morphine withdrawal syndrome were evaluated by an observer blind to genotype. Morphine withdrawal global score was calculated using the relative weight values for behavioral signs previously described in Maldonado et al. (1996); a weight value of 0.5 was used for those signs that were not included in that study. Morphine withdrawal-induced CPA: Morphine-induced CPA was evaluated in the same four black acrylic conditioning boxes previously described using interchangeable grid and hole floors as CS. Half of the subjects of each genotype/treatment group experienced morphine-withdrawal (see below) in each of these alternative floors (CS+). The conditioning was achieved in a single conditioning session. Thus, after receiving the drug regime previously described, $2 \mathrm{~h}$ after the last morphine injection (day 6), morphine-dependent mice received a subcutaneous naloxone challenge $(0.1 \mathrm{mg} / \mathrm{kg})$. Immediately after this treatment, mice were placed for $15 \mathrm{~min}$ in a conditioning box equipped with one of the floors, arbitrarily assigned as CS+. Twenty-four hours after this conditioning session, the possible development of CPA was evaluated by a single preference test (duration: $15 \mathrm{~min}$ ). In this case the floor of each conditioning chamber was divided (half grid, half holes) and the time spent by each mouse in each type of floor was assessed by a video-tracking system (SMART, Panlab S.L; Barcelona, Spain). Similarly to the CPP experiment previously described, the percent of time spent in the CS+ was used as the index of the conditioned avoidance.

\section{OTHER TECHNIQUES}

Nissl staining, in situ hybridization and immunostaining were performed as previously described (Lopez de Armentia et al., 2007; Jancic et al., 2009). Anti Flag-M2 (F1804, mouse monoclonal) and anti-DARPP-32 (ab40801, rabbit monoclonal) antibodies were obtained from Abcam (Cambridge, UK).

\section{STATISTICS}

Data were normalized by squared root transformation in all cases to ensure a normal distribution and allow the use of percentages in parametric statistics. The different indexes of the general behavioral characterization conducted in ACREB-St and their corresponding littermate controls were analyzed by a multiple analysis of variance (MANOVA) using the genotype as between groups factor. CPP and CPA were analyzed by means of separate two-way ANOVA (genotype $\times$ treatment) using as dependent variable the percent of time in the CS+. For clarity, CPA data are displayed as an arithmetic transformation of this variable (\% time spent in CS+ minus 
100\%). Morphine withdrawal was analyzed by means of MANOVA using the genotype as a between-groups factor. The Chi-squared test was used to compare the proportion of mice exhibiting ptosis or piloerection in each group at any moment of the morphine withdrawal test.

\section{RESULTS \\ REGULATED AND HIGHLY RESTRICTED EXPRESSION OF A-CREB IN STRIATUM}

To investigate the consequences of impaired CREB-dependent gene expression in striatal neurons, we generated transgenic mice expressing the repressor of CRE-binding activity A-CREB using the CamKII $\alpha$-tTA/tetO system of inducible double transgenics (Mayford et al., 1996). One of these bitransgenic strains (resulting of crossing tetO-A-CREB line AC100) showed expression of A-CREB transcripts exclusively in striatal neurons (Figures 1A-C), for this reason, we will refer to it as ACREB-St mice. This strain might be especially appropriate to investigate the role of CREBdependent gene expression in striatal-related function and behavior. Histological examination of brain sections did not reveal any gross abnormality in ACREB-St mice (Figure 2A), whereas immunohistological analyses revealed the expression of this inhibitory peptide in neurons of both the dorsal hippocampus and the nucleus accumbens
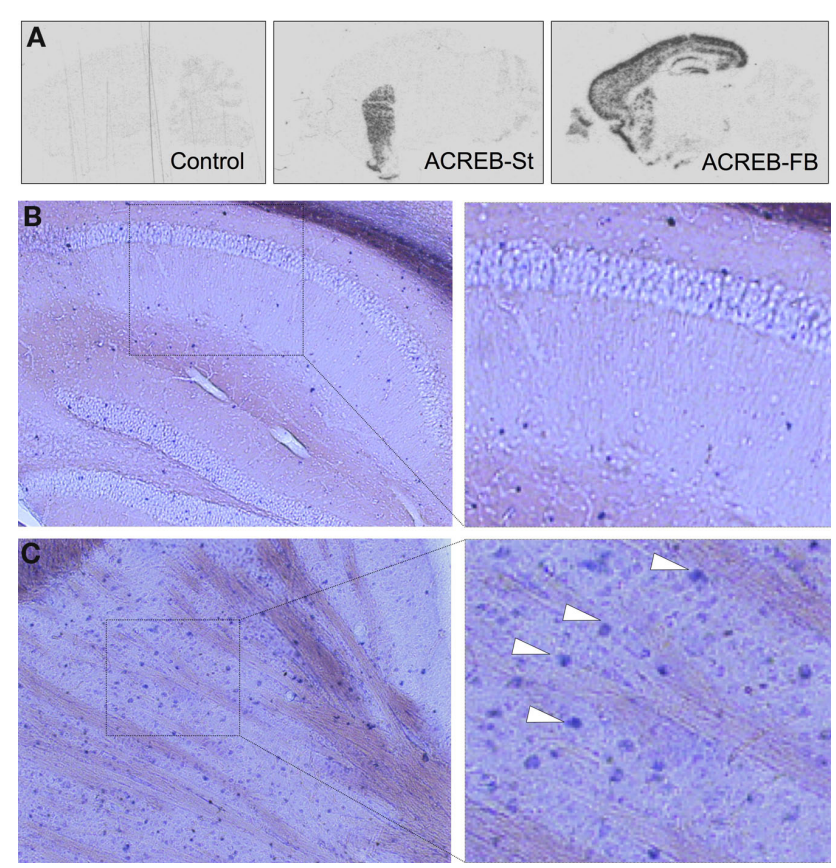

FIGURE 1 | Bitransgenic mice with inducible and highly restricted expression of A-CREB in striatal neurons. (A) Comparison of the pattern of transgene expression in brain sagittal sections from a bitransgenic ACREB-St mouse, a control littermate, and a bitransgenic ACREB-FB mouse (the strain characterized in detail in Jancic et al., 2009) visualized by in situ hybridization using an oligonucleotide probe specific for the A-CREB transgene. (B,C) DIG in situ hybridization showing transgene expression in the striatum (C), but not in the hippocampus (B) of a 5-months old ACREB-St mouse using a probe specific for A-CREB transgene. The white arrows label positive striatal cells. The same pattern of expression was observed in three mice per genotype.
(NA, Figure 2B,C). Double immunostaining with antibodies against Flag-M2, which recognizes A-CREB, and DARPP-32, the most commonly used marker for medium spiny neurons, demonstrated that $100 \%$ of the neurons expressing the transgene belong to this neuronal type (Figure 3).
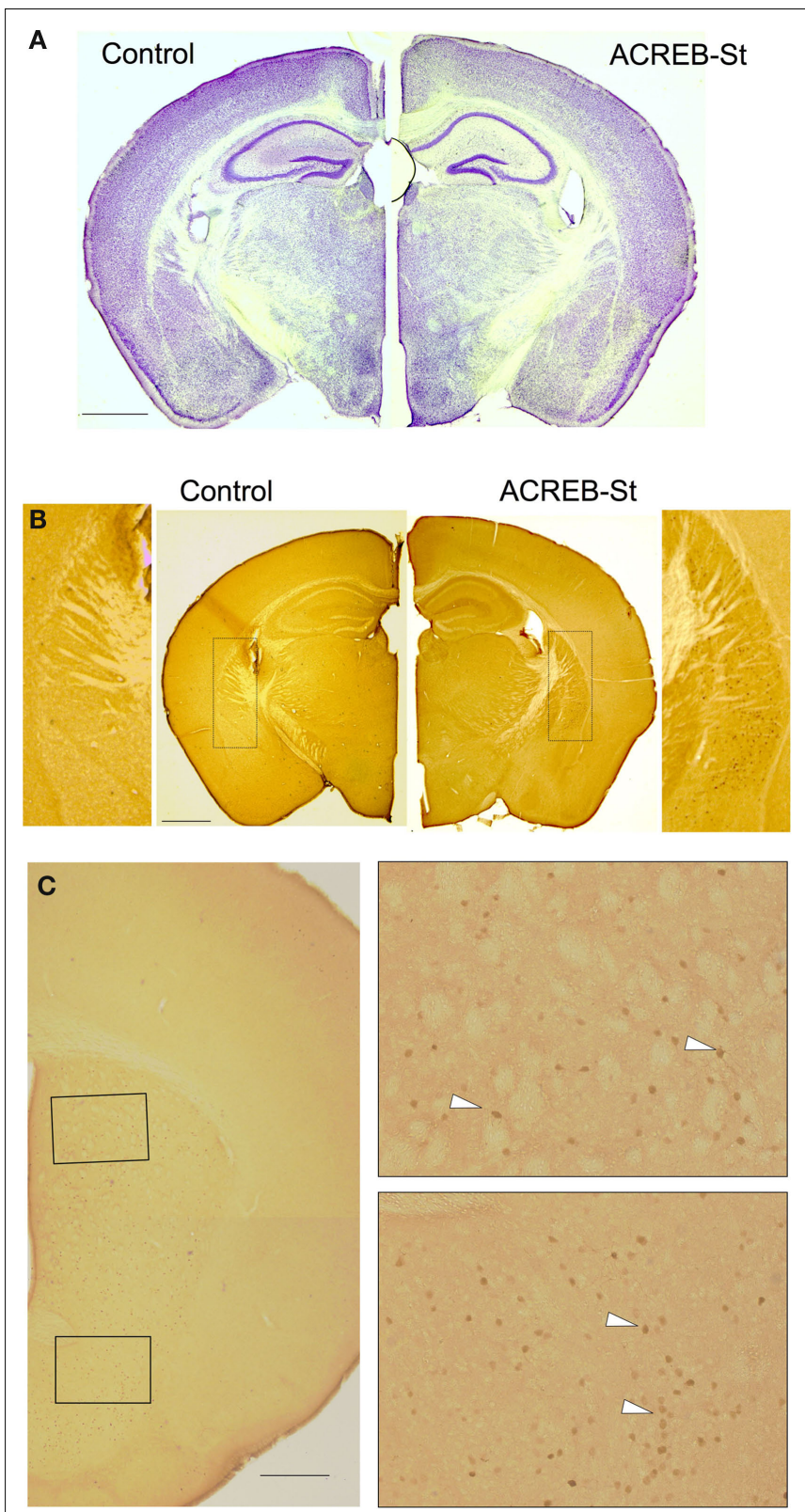

FIGURE 2 | Normal gross brain anatomy in ACREB-St mice. (A) Nissl staining of the coronal brain section of an adult ACREB-St mouse and a control littermate did not reveal gross anatomical changes (scale bar: $1 \mathrm{~mm}$ ). (B) Immunostaining of brain sections showed Flag-M2 immunoreactivity in the dorsal striatum of ACREB-St mice (right), but not in their control littermates (left). Hippocampus, cortical layers and other brain regions did not show immunoreactivity (scale bar: $1 \mathrm{~mm}$ ). (C) Immunostaining of coronal brain sections in an ACREB-St mouse showed positive cells (white arrows) both in the dorsal striatum (upper inset) and in the nucleus accumbens (lower inset). Scale bar: $0.5 \mathrm{~mm}$. The same pattern of expression was observed in three mice per genotype. 

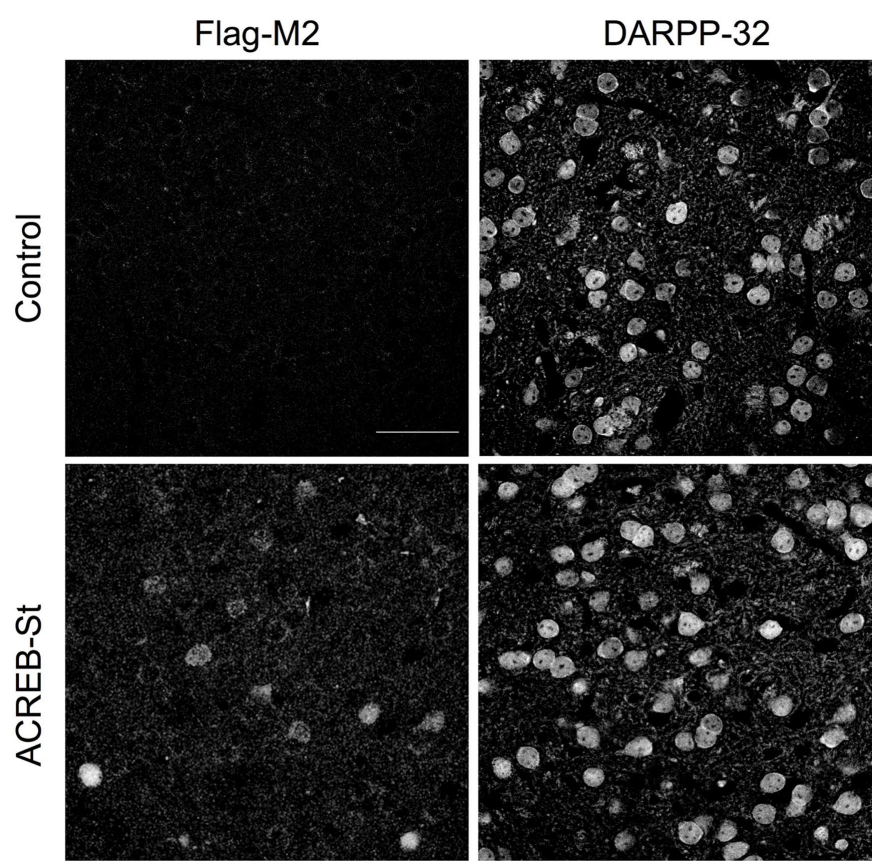

Flag-M2/DARPP-32/DAPI
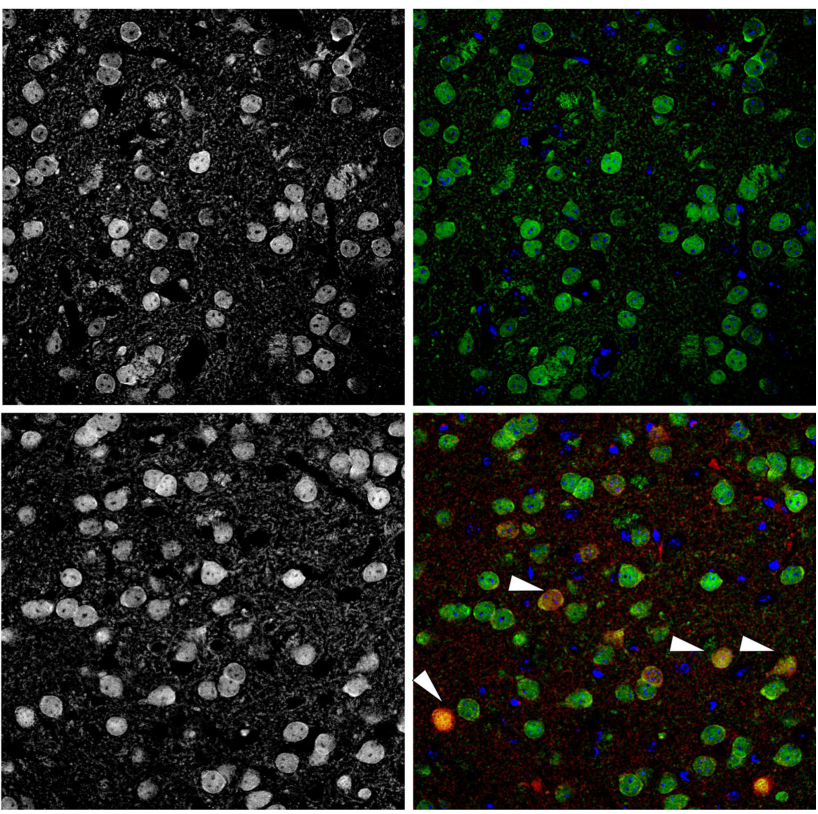

FIGURE 3 | Expression of A-CREB protein and colocalization with a marker for interneurons. Double immunostaining using antibodies against Flag-M2, which recognizes A-CREB, and DARPP-32. The colocalization of both markers demonstrates that the transgene is expressed in striatal GABAergic medium spiny neurons (MSNs). The right panels show the overlay of Flag-M2 (in red) and DARPP-32 (in green) staining, and DAPI (in blue) counterstaining. The white arrows label positive MSNs for Flag-M2 and DARPP-32 (in yellow). Scale bar: $50 \mu \mathrm{m}$. The same result was observed in three mice per genotype.

\section{GENERAL BEHAVIORAL CHARACTERIZATION}

Although a MANOVA comparing both strains of mice yielded a genotype effect (Wilk's lambda 7, $8=0.125, p<0.01$ ), follow up ANOVA-based univariate comparisons revealed that with the exception of a slight decrease of body weight $(p<0.01)$, ACREB-St mice did not display any major morphologic or developmental alteration. Similarly, at the behavioral level, ACREB-St mice did not exhibit major alterations regarding spontaneous locomotion, novelty reactivity or anxiety-like behavior. Thus, ACREB-St mice and their control littermates did not differ in the distance traveled in the open field or the number of rearing episodes in the novel cage test. Also, both genotypes spent similar amounts of time in the open arms of the plus maze and in the light compartment of the light-dark box, although in these tests ACREB-St mice displayed a reduction in the total number of arms entries and compartment visits ( $p<0.01$ in all cases). These results are shown in Table 1.

\section{MORPHINE-INDUCED CONDITIONED PLACE PREFERENCE}

Regardless their genotype or the diet they received, mice did not display innate preference for any of the compartments of the conditioning box during the habituation session $\left(F_{1,30}=0.42\right.$, N.S. $)$, whereas repeated pairings of morphine to a specific environment resulted in an increase of preference for that environment during a test conducted at later times, a behavior referred as conditioned place preference or CPP (see scheme in Figure 4A). As shown in Figure 4B, the strength of the CPP test carried out $24 \mathrm{~h}$ after the last pairing differed among genotypes in mice in which transgene expression was turned on (Figure 4B: Off Dox). The addition of dox to the mouse diet can repress transgene expression in less than
Table 1 | General characterization of ACREB-St mice.

\begin{tabular}{lcc}
\hline & Control & ACREB-St \\
\hline Body weight & $31.5 \pm 0.72$ & $25.3 \pm 0.8^{*}$ \\
Locomotion (cm/20 min) & $3517.4 \pm 289.5$ & $3721.2 \pm 362.7$ \\
Novelty reaction (rearing & $34.2 \pm 4.1$ & $39.2 \pm 3.9$
\end{tabular}

episodes in $5 \mathrm{~min}$ )

\section{PLUS MAZE}

Time spent in the open

$29.85 \pm 10.66$

$40.28 \pm 12.93$

arms (s)

Total number of entries

$13.71 \pm 1.58$

$6.78 \pm 0.85^{*}$

\section{DARK-LIGHT BOX}

Time spent in the light

$91.53 \pm 21.38$

$57.08 \pm 7.42$

compartment (s)

Total number of

$9.71 \pm 0.52$

$6.22 \pm 0.91 *$

compartment changes

Body weight and performance in several basal behavioral tests were assessed in a cohort of nine ACREB-St mice and seven control littermates. Data are presented as mean \pm SEM. ( $\left.^{*}\right)$ Indicates $p<0.01$.

1 week (Jancic et al., 2009). Notably, dox treatment abolished the differences between genotypes in the CPP task (Figure 4B: On Dox). Thus, a two-way ANOVA (genotype $\times$ treatment) revealed a significant interaction between both factors $\left(F_{1,33}=4.39, p<0.05\right)$ and post hoc comparisons showed that dox-untreated ACREB-St mice significantly differed of the rest of the experimental groups $(p<0.05$ in all cases), demonstrating the reversal of the CPP phenotype. 


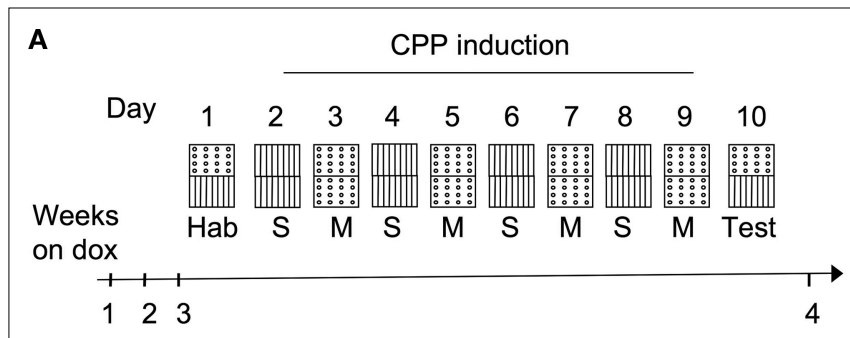

B

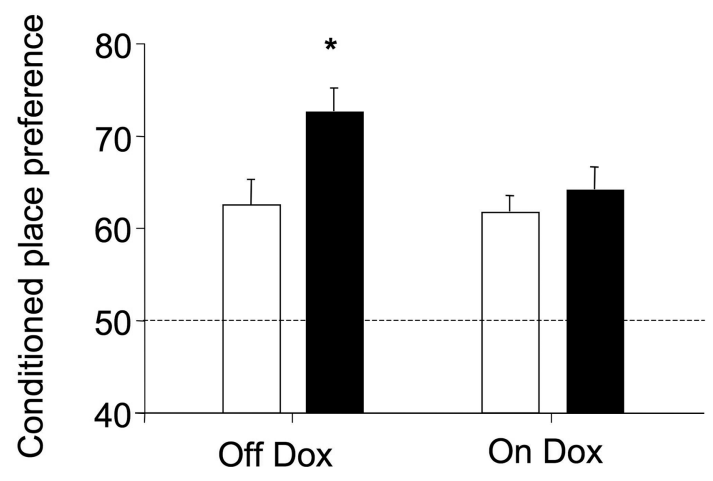

FIGURE 4 | Conditioned place preference. (A) Schematic representation of the behavioral training protocol and treatment groups used in the conditioned place preference (CPP) experiment. Although for clarity the scheme presents the "holes" floor as CS+, this factor was counterbalanced across genotypes and dox treatment (see Section "Materials and Methods" for further details). (B) CPP was evaluated as the percent of time spent in the environment paired to morphine $(20 \mathrm{mg} / \mathrm{kg}$ ) administration during a preference test conducted $24 \mathrm{~h}$ after the last conditioning session. Black and white bars depict the results for ACREB-St and littermate control mice, respectively. The horizontal dotted line indicates the indifference (e.g., 50\%) point. ${ }^{*} p<0.05$ as compared to all other groups.

Table 2 | Morphine withdrawal signs in ACREB-St mice and control littermates.

\begin{tabular}{lcc}
\hline & Control $(\boldsymbol{N}=\mathbf{7})$ & ACREB-St $(\boldsymbol{N}=\mathbf{9})$ \\
\hline Forepaw tremor & $11.00 \pm 2.39$ & $8.11 \pm 1.34$ \\
Shakes & $3.43 \pm 0.68$ & $4.00 \pm 0.74$ \\
Chewing & $5.43 \pm 0.92$ & $4.44 \pm 0.60$ \\
Jumping & $21.57 \pm 3.51$ & $26.33 \pm 2.76$ \\
Grooming & $12.43 \pm 2.74$ & $12.88 \pm 2.45$ \\
Rearing & $19.43 \pm 2.75$ & $10.22 \pm 1.07^{*}$ \\
Digging & $10.14 \pm 2.72$ & $3.89 \pm 0.65^{*}$ \\
Ptosis & $4 / 7$ & $5 / 9$ \\
Piloerection & $5 / 7$ & $5 / 9$ \\
Global withdrawal score & $59.30 \pm 2.48$ & $52.96 \pm 4.36$ \\
\hline
\end{tabular}

Morphine-dependent mice were challenged with a single naloxone $10.1 \mathrm{mg} / \mathrm{kg}$; $I P)$ injection and the frequency of different signs of morphine withdrawal was measured. Data are expressed as mean \pm SEM, with the exception of ptosis and piloerection which are expressed as the proportion of subjects exhibiting those signs in each genotype. $\left(^{*}\right)$ indicates $p<0.05$.

\section{MORPHINE WITHDRAWAL}

The severity of morphine withdrawal did not substantially differ between ACREB-St mice and their control littermates (Table 2). A MANOVA comparing the frequency of different morphine withdrawal signs, such as forepaw tremor, shakes, chewing, jumping grooming, rearing and digging, did not yield a significant difference between genotypes (Wilk's lambda ${ }_{7,8}=0.303, p=0.10$ ). Specific comparisons conducted by independent one-way ANOVAs mainly confirmed this overall result, although also revealed a reduced number of rearing $(p<0.01)$ and digging $(p<0.05)$ episodes in ACREB-St mice. Further, the proportion of mice exhibiting ptosis or piloerection did not differ between genotypes when compared by means of the chi-squared test ( $p=0.61$ and 0.95 , respectively). Similar conclusions, that is, the lack of differences between genotypes in the intensity of morphine withdrawal were drawn using a global withdrawal score $\left(t_{14}=1.16, p=0.26\right)$.

MORPHINE WITHDRAWAL-INDUCED CONDITIONED PLACE AVOIDANCE

Regardless of their genotype or the presence of dox in the diet, mice did not display any innate preference for any of the compartments of the conditioning box during the habituation session $\left(F_{1,30}=0.42\right.$, N.S. $)$. However, pairing morphine withdrawal with an environment resulted in the avoidance of this environment during a test conducted $24 \mathrm{~h}$ later (CPA, see scheme in Figure 5A). The magnitude of this effect markedly differed among the different treatment groups. Thus, as revealed by a two-way ANOVA (genotype $\times$ diet), both main factors $\left(F_{1,30}=29.15, p<0.01\right.$ and

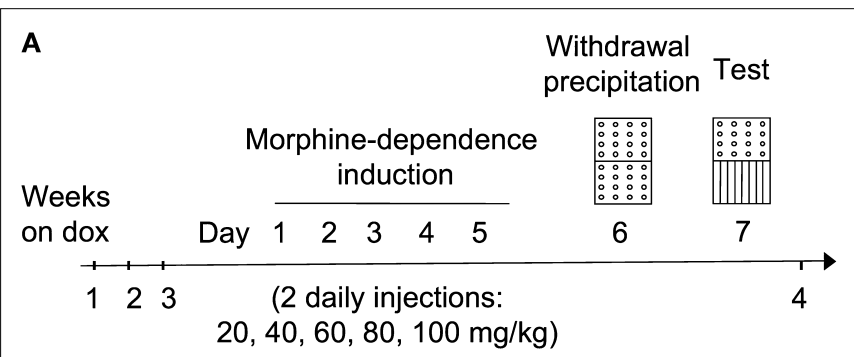

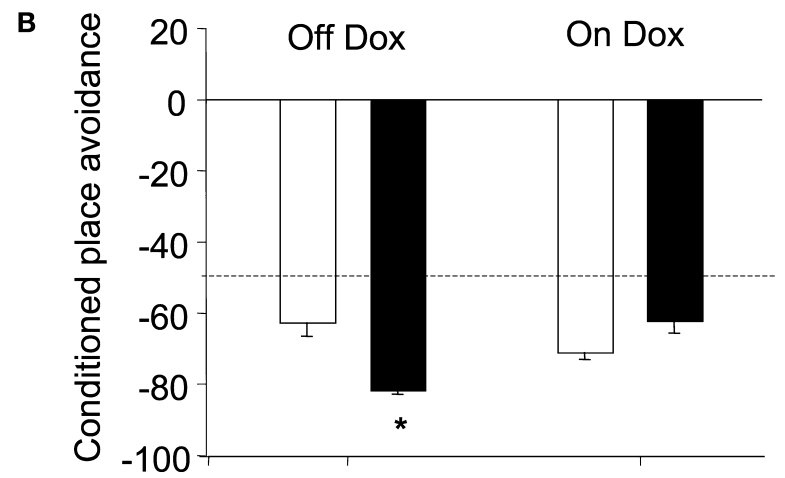

FIGURE 5 | Conditioned place avoidance. (A) Scheme depicting the behavioral training and treatment groups used in the conditioned place avoidance (CPA) procedure. Although for clarity the scheme presents the "holes" floor as CS+, this factor was counterbalanced across genotypes and dox treatment (see Section "Materials and Methods" for further details). (B) CPA was evaluated as the percent of time spent in the environment paired to morphine withdrawal during a preference test conducted $24 \mathrm{~h}$ after the last conditioning session but, for clarity, those data were arithmetically transformed (CPA = \% time spent in CS+ minus 100\%). The black and white bars depict the results for ACREB-St and littermate control mice, respectively. The horizontal dotted line indicates the indifference (e.g., 50\%) point. ${ }^{*} p<0.05$ as compared to all other groups. 
$F_{1,30}=4.43, p<0.05$, respectively) as well as its interaction yielded a significant effect $\left(F_{1,30}=5.07, p<0.05\right)$. Thus, as it can be seen in Figure 5B, ACREB-St mice off dox (transgene on) spent less time than their control littermates in the environment associated to morphine withdrawal. Again, post hoc comparisons revealed that dox-untreated ACREB-St mice exhibit a higher degree of CPA than the other three groups ( $p<0.05$ in all cases), demonstrating that CREB inhibition enhanced the avoidance behavior associated to withdrawal in a reversible manner.

\section{DISCUSSION}

In the present study we introduce a new strain of bitransgenic mice, ACREB-St, expressing the repressor of the CREB family of transcription factors A-CREB in striatal neurons in a regulatable manner. ACREB-St mice did not show any gross anatomical alteration or apparent neurodegeneration. These results suggest that this strain of mice might be a more suitable tool to study the role of CREB in striatal neurons than some previous genetic models that lacked striatal-specificity (Maldonado et al., 1996; Valverde et al., 2004) or presented some major physiological and morphological alterations, such as striatal degeneration (Mantamadiotis et al., 2002). The use of genetically modified mice might also provide some advantages over viral-mediated delivery, such as being a less intrusive procedure (Carlezon et al., 1998; Barrot et al., 2002), although this may be achieved at the prize of a lower anatomical resolution.

ACREB-St mice exhibited a dox-reversible enhancement of CPP after repeated morphine administration. This observation is in agreement with previous studies concluding that an increase of CREB activity in the NA results in a decrease of drug-induced CPP, whereas the blockade of CREB function through expression of a dominant-negative protein had the opposite effect (Carlezon et al., 1998; Barrot et al., 2002; McClung and Nestler, 2003). However, the role of CREB in the striatum does not seem to be restricted to appetitive contingencies. Despite showing normal withdrawal, ACREB-St also displayed a dox-reversible enhancement of conditioned avoidance to a context paired to morphine withdrawal. This observation is in agreement with previous studies showing that viral-mediated overexpression of $\mathrm{MCREB}$ (another dominant negative CREB mutant) in the shell of the NA increased CPA induced by high doses of naloxone in morphine-naïve rats, whereas CREB overexpression resulted in the opposite effect (Barrot et al., 2002). Studies involving the overexpression of the natural CREB repressor ICER in striatal areas like the NA also resulted in enhanced responses toward both appetitive and aversive stimuli (Green et al., 2006). Thus, CREB-related neuroplasticity in some striatal regions seems to dampen behavioral responses to emotionally relevant stimuli regardless its appetitive or aversive value.

Previous studies using CREB deficient mice have reported a decrease of the intensity of morphine withdrawal (Maldonado et al., 1996; Valverde et al., 2004), this finding was not reproduced in ACREB-St mice, but such a result was not unexpected because the implication of CREB in the somatic and physiological signs of morphine withdrawal has been directly related to the activity of this transcription factor in the locus coeruleus and not to its action in striatal structures (Valverde et al., 2004; Han et al., 2006). Conversely, the fact that the expression of this transcription factor in the striatum substantially contributes to the magnitude of approach/ avoidance responses toward CS seems in agreement with the role of this anatomical structure in the regulation of several cognitive and motor functions (Everitt and Robbins, 2005). Our findings and those of some previous studies (Carlezon et al., 1998; Barrot et al., 2002; McClung and Nestler, 2003; Green et al., 2006) could be interpreted as the result of inhibiting CREB activity at the NA. In this regard, it is known that a reduction of CREB activity in GABAergic neurons of the NA decreases their excitability (Dong et al., 2006). The reduction in the firing of GABAergic neurons would result in decreased GABA and dynorphin-mediated inhibition over VTAdopaminergic neurons (Carlezon et al., 2005) and this reduction of the inhibitory input would, in turn, lead to an increased probability of firing of dopaminergic VTA neurons as well as increased dopamine release and dopamine-receptor binding at the level of the NA. Interestingly, both a reduction in the number of accumbal firing neurons (German and Fields, 2007) as well as in the magnitude of the dopaminergic input to the NA core have been directly related to the acquisition and consolidation of Pavlovian approach responses (Parkinson et al., 1999; Di Ciano et al., 2001; Everitt and Robbins, 2005). However, since the reduction on CREB-related gene expression in ACREB-St mice extends to striatal areas other than the NA (i.e., dorsal striatum, Figures 1 and 2), alternative explanations could also be considered to explain the mechanisms underlying the effects on behavior presented here (see Fasano et al., 2009). Thus, disinhibition of striatal GABAergic interneurons does not only lead to a disinhibition of the VTA dopaminergic neurons projecting to the NA, but also to those connecting the substantia nigral and dorsal regions of the striatum. Several regions of the striatum might mediate behavioral processes relevant for the observed phenotypes (Kelley, 2004; Everitt and Robbins, 2005).

In summary, the results of the present study suggest that CREBrelated neuroplasticity in the striatum might be implicated in the promotion of appropriate avoidance or approach responses toward environmental stimuli associated to emotionally relevant stimuli. The exact mechanisms underlying this process are unclear, but coupled to data from previous studies, our results indicate that those behavioral findings might arise from the ability of CREB-mediated neuroplasticity to alter dopamine-related mechanisms at the NA and/or dorsal striatum regions. Further, as our results differ from those obtained when using anatomically unspecific CREB deficient mice (Valverde et al., 2004; Bilbao et al., 2008), but are coincident with those promoting a selective reduction of CREB-related activity in the NA (Carlezon et al., 1998; Barrot et al., 2002; McClung and Nestler, 2003; Green et al., 2006), the present study emphasizes the importance of using experimental complementary approaches suitable to dissect the multiple actions of CREB in different brain regions.

\section{ACKNOWLEDGMENTS}

We thank Roman Olivares, Alicia Dosda y Gema Caballer for excellent technical assistance. Carles Sanchis-Segura holds a Ramón y Cajal contract from Ministerio de Ciencia e Innovación. Research at Carles Sanchis-Segura's lab was supported by a Grant of Ministerio de Ciencia e Innovación (PSI2-8-01317/PSIC) and a grant from Fundación Eugenio Rodriguez Pascual. Research at Angel Barco's lab was supported by the European Commission grant MEXT-CT-2003-509550 and the Spanish Ministry of Science and Innovation Grants SAF2008-00611 and CSD2007-00023. 


\section{REFERENCES}

Barrot, M., Olivier, J. D., Perrotti, L. I., DiLeone, R. J., Berton, O., Eisch, A. J., Impey, S., Storm, D. R., Neve, R. L., Yin, J. C., Zachariou, V., and Nestler, E. J. (2002). CREB activity in the nucleus accumbens shell controls gating of behavioral responses to emotional stimuli. Proc. Natl. Acad. Sci. USA 99, 11435-11440.

Bilbao, A., Parkitna, J. R., Engblom, D., Perreau-Lenz, S., Sanchis-Segura, C., Schneider, M., Konopka, W., Westphal, M., Breen, G., Desrivieres, S., Klugmann, M., Guindalini, C., Vallada, H., Laranjeira, R., de Fonseca, F. R., Schumann, G., Schutz, G., and Spanagel, R. (2008). Loss of the $\mathrm{Ca}^{2+} /$ calmodulin-dependent protein kinase type IV in dopaminoceptive neurons enhances behavioral effects of cocaine. Proc. Natl. Acad. Sci. USA 105, 17549-17554.

Carlezon, W. A. Jr., Duman, R. S., and Nestler, E. J. (2005). The many faces of CREB. Trends Neurosci. 28, 436-445.

Carlezon, W.A. Jr., Thome, J., Olson, V. G., Lane-Ladd, S. B., Brodkin, E. S., Hiroi, N., Duman, R. S., Neve, R. L., and Nestler, E. J. (1998). Regulation of cocaine reward by CREB. Science 282, 2272-2275.

Di Ciano, P., Cardinal, R. N., Cowell, R. A., Little, S. J., and Everitt, B. J. (2001). Differential involvement of NMDA, AMPA/kainate, and dopamine receptors in the nucleus accumbens core in the acquisition and performance of pavlovian approach behavior. J. Neurosci. 21, 9471-9477.

Domjan, M. (2005). Pavlovian conditioning: a functional perspective. Annu. Rev. Psychol. 56, 179-206.

Dong, Y., Green, T., Saal, D., Marie, H., Neve, R., Nestler, E. J., and Malenka, R. C. (2006). CREB modulates excitability of nucleus accumbens neurons. Nat. Neurosci. 9, 475-477.

Everitt, B. J., and Robbins, T. W. (2005). Neural systems of reinforcement for drug addiction: from actions to habits to compulsion. Nat. Neurosci. 8, 1481-1489.

Fasano, S., Pittenger, C., and Brambilla, R. (2009). Inhibition of CREB activity in the dorsal portion of the striatum potentiates behavioral responses to drugs of abuse. Front. Behav. Neurosci. doi: 10.3389/neuro.08.029.2009

German, P. W., and Fields, H. L. (2007). Rat nucleus accumbens neurons persistently encode locations associated with morphine reward. J. Neurophysiol. 97, 2094-2106.

Green, T.A., Alibhai, I. N., Hommel, J. D., DiLeone, R. J., Kumar, A., Theobald, D. E., Neve, R. L., and Nestler, E. J. (2006). Induction of inducible cAMP early repressor expression in nucleus accumbens by stress or amphetamine increases behavioral responses to emotional stimuli. J. Neurosci. 26, 8235-8242.

Han, M. H., Bolanos, C. A., Green, T. A, Olson, V. G., Neve, R. L., Liu, R. J., Aghajanian, G. K., and Nestler, E. J. (2006). Role of cAMP response element-binding protein in the rat locus ceruleus: regulation of neuronal activity and opiate withdrawal behaviors. J.Neurosci. 26, 4624-4629.

Hyman, S. E., Cole, R. L., Konradi, C., and Kosofsky, B. E. (1995). Dopamine regulation of transcription factor-target interactions in rat striatum. Chem. Senses 20, 257-260.

Jancic, D., Lopez de Armentia, M., Valor, L. M., Olivares, R., and Barco, A. (2009). Inhibition of cAMP response element-binding protein reduces neuronal excitability and plasticity, and triggers neurodegeneration. Cereb. Cortex. [Epub ahead of print].

Kelley, A. E. (2004). Ventral striatal control of appetitive motivation: role in ingestive behavior and reward-related learning. Neurosci. Biobehav. Rev. 27, 765-776.

Konradi, C., Cole, R. L., Heckers, S., and Hyman, S. E. (1994). Amphetamine regulates gene expression in rat striatum via transcription factor CREB. J. Neurosci. 14, 5623-5634.

Lopez de Armentia, M., Jancic, D., Olivares, R., Alarcon, J. M., Kandel, E. R., and Barco, A. (2007). cAMP response element-binding protein-mediated gene expression increases the intrinsic excitability of
CA1 pyramidal neurons. J. Neurosci. 27, 13909-13918.

Maldonado, R., Blendy, J. A., Tzavara, E. Gass, P., Roques, B. P., Hanoune, J., and Schutz, G. (1996). Reduction of morphine abstinence in mice with a mutation in the gene encoding CREB Science 273, 657-659.

Mantamadiotis, T., Lemberger, T., Bleckmann, S. C., Kern, H. Kretz, O., Martin Villalba, A., Tronche, F., Kellendonk, C., Gau, D. Kapfhammer, J., Otto, C., Schmid, W., and Schutz, G. (2002). Disruption of CREB function in brain leads to neurodegeneration. Nat. Genet. 31, 47-54.

Mayford, M., Bach, M. E., Huang, Y. Y. Wang, L., Hawkins, R. D., and Kandel, E. R. (1996). Control of memory formation through regulated expression of a CaMKII transgene. Science 274, 1678-1683.

Mayr, B., and Montminy, M. (2001). Transcriptional regulation by the phosphorylation-dependent factor CREB. Nat. Rev. Mol. Cell Biol. 2, 599-609.

McClung, C. A., and Nestler, E. J. (2003). Regulation of gene expression and cocaine reward by CREB and DeltaFosB. Nat. Neurosci. 6 , 1208-1215.

McClung, C. A., and Nestler, E. J. (2008). Neuroplasticity mediated by altered gene expression. Neuropsychopharmacology 33, 3-17.

Nestler,E.J.(2004).Molecular mechanisms of drug addiction. Neuropharmacology 47(Suppl. 1), 24-32.

Olson, V.G.,Zabetian, C. P., Bolanos, C. A. Edwards, S., Barrot, M., Eisch, A. J., Hughes, T., Self, D. W., Neve, R. L., and Nestler, E. J. (2005). Regulation of drug reward by cAMP response element-binding protein: evidence for two functionally distinct subregions of the ventral tegmental area. J. Neurosci. 25, 5553-5562.

Parkinson, J. A., Olmstead, M. C. Burns, L. H., Robbins, T. W., and Everitt, B. J. (1999). Dissociation in effects of lesions of the nucleus accumbens core and shell on appetitive pavlovian approach behavior and the potentiation of conditioned reinforcement and locomotor activity by D-amphetamine. J. Neurosci. 19, 2401-2411

Sanchis-Segura, C., Borchardt, T., Vengeliene, V., Zghoul, T., Bachteler, D., Gass, P., Sprengel, R., and Spanagel, R. (2006). Involvement of the AMPA receptor GluR-C subunit in alcohol-seeking behavior and relapse. J. Neurosci. 26, 1231-1238.

Shaw-Lutchman, TZ, Impey, S., Storm, D., and Nestler, E. J. (2003). Regulation of CRE-mediated transcription in mouse brain by amphetamine. Synapse 48, 10-17.

Valverde, O., Mantamadiotis, T., Torrecilla, M., Ugedo, L., Pineda, J., Bleckmann, S., Gass, P., Kretz-O, Mitchell, J. M., Schutz, G., and Maldonado, R. (2004). Modulation of anxiety-like behavior and morphine dependence in CREB-deficient mice. Neuropsychopharmacology 29, 1122-1133.

Walters, C. L., Kuo, Y. C., and Blendy, J. A. (2003). Differential distribution of CREB in the mesolimbic dopamine reward pathway. J. Neurochem. 87, 1237-1244

Conflict of Interest Statement: The authors declare that the research was conducted in the absence of any commercial or financial relationships that could be construed as a potential conflict of interest.

Received: 05 May 2009; paper pending published: 17 June 2009; accepted: 24 August 2009; published online: 08 September 2009.

Citation: Sanchis-Segura C, Jancic D, Jimenez-Minchan M and Barco A (2009) Inhibition of $c A M P$ responsive element binding protein in striatal neurons enhances approach and avoidance responses toward morphine-and morphine withdrawalrelated cues. Front. Behav. Neurosci. 3:30. doi: 10.3389/neuro.08.030.2009

Copyright (C) 2009 Sanchis-Segura, Jancic, Jimenez-Minchan and Barco. This is an open-access article subject to an exclusive license agreement between the authors and the Frontiers Research Foundation, which permits unrestricted use, distribution, and reproduction in any medium, provided the original authors and source are credited. 